\title{
Time for a Change? A Research Update and Pilot Study Results on Academic Time-Based Decision-Making
}

\author{
Nicholas J. Rupar and David S. Strong \\ Queen's University \\ nicholas.rupar@queensu.ca, david.strong@queensu.ca
}

\begin{abstract}
Engineering students in Canada and around the world are facing numerous challenges with their time, including absenteeism from class, surface approach to learning, and high stress levels. A research study to understand the time management habits of engineering students is ongoing and a pilot study has been completed. This paper contains an overview of the relevant background theories, the application of these theories to a new survey instrument, and the pilot study used to test and improve this instrument.
\end{abstract}

The new survey instrument on time management and decision making was required as existing instruments published in the literature were considered to be flawed and inadequate for this study. The new instrument incorporates a decision-making dimension following the think-plan-do models of self-regulated learning theories. Analysis of this instrument will assume clusters, rather than factors, of time management behaviours to be the basis for grouping individuals.

The pilot study was conducted on a number of selfselected graduate engineering students in November 2019. Participants filled out an online survey and then some volunteered for a think-aloud interview. Changes resultant from the pilot study analysis included question modifications, Likert scale modifications, and user experience improvements. The pilot study resulted in an overall improvement to the validity, reliability, and completeness of the survey instrument.

The full study is currently being administered to undergraduate engineering students. The results will be published to help inform the manner in which time management is taught and used by engineering students.

Keywords: Time Management, Decision Making, Self-Regulated Learning, Self-Efficacy, Interventions, Mental Well-being.

\section{Introduction}

"How sour sweet music is,

When time is broke and no proportion kept!

So is it in the music of men's lives."

- Shakespeare, Richard II

From Canada to Australia, the United States, and Europe, engineering students appear to be struggling with how to spend their time while in school [1]-[7]. Over the last five years in Canada, there has been an apparent increase in instructor-reported surface learning and class absences, as well as student-reported stress, perceived workload, and mental wellness issues [7]-[11]. All these issues may be linked to students' ability to manage their time effectively. At the same time, conventional perceptions of time and conventional time management wisdom is being challenged as ineffective in describing a wide variety of possibly advantageous time-management strategies [12]-[16].

A research study has been designed to explore the extent to which engineering student time management and decision making skills are connected to these issues by asking three questions: (1) What is the diversity of engineering student time management and decision making behaviours? (2) What are the consequences of different behaviours on academics and non-academics? (3) What are the causes of such behaviours?

An increased understanding of student time management may lead to improved advice, training, or systemic changes that benefit students by removing barriers and promoting effective behaviours. This in turn may result in more effective and efficient teaching and learning practices.

This paper will first summarize the literature in time perceptions, time management, and decision making, before briefly describing the research instrument. It will then detail the pilot study undertaken to test the instrument and the benefits that the pilot provided.

\section{Literature Review and Theory}

A relatively extensive literature review and introduction to the concept of "Academic Time-Based Decision Making" (ATBDM) has been discussed in a prior paper [1]. ATBDM was described as encompassing both time management and decision making in an academic context. This concept was reinforced by a review of various theories of time, time management, and decision making, and an overview of the existing situation and attempted interventions.

At that time, it was suggested that studying the factors affecting student ATBDM from a personal and institutional level would be most beneficial. Although this is still an intention of the study, an examination of the time management literature exposed a lack of appropriate research instruments with which to simultaneously measure time management and decision making [15][17]. A new survey tool was therefore required.

CEEA20; Paper 158

Montreal; June 18-20, 2020 
This section will briefly review the motivations to study ATBDM, before summarizing the relevant theories and resultant instrument creation process.

\subsection{Motivation for Studying Time Management Behaviours}

"Why study time management in students instead of looking at the deeper systemic issues in engineering education?" In fact, there is some indication that time management skills may be both indicator and cause of many issues currently facing engineering students, including surface learning, attendance, stress levels, and cheating.

Surface learning, where engineering students prioritize the completion of a deliverable over learning the material therein, has been observed by professors and implied by students in several recent Canadian studies [3], [8], [10]. In the United States, it has been shown that engineering students under time stress will switch to coping methods of learning, which appear to resemble surface-learning behaviours [18].

Lack of attendance in lectures, distractions in class, and lateness has also been observed. Reasons for this both speculated and documented include non-ideal scheduling (e.g. perceived 'early' classes), finishing other work (i.e. prioritizing other classes), assuming the material can be learned elsewhere, and taking personal time [2], [7]. Personal time includes time to be social and time to take a mental health break, both of which may be an attempt to reclaim a sense of control over time. Being late to class, for example by finishing other homework, may also indicate alternative or poor time management strategies used by students.

Homework completion rates in American engineering students has been linked to having more hours of external commitments [6]. There is some indication that Canadian engineering students are now involved with more extracurriculars, which may indicate one possible cause [19], [20]. Promisingly, in areas of homework, extracurricular involvement (specifically design teams), and online courses, engineering educators have with some success alleviated time management pressure on students through course design [6], [19], [21].

Cheating, including copying homework, has been recently observed in Canadian engineering students [8]. Results in Smith and Maw's study imply that some degree of copying is done because of students being under time pressure.

Finally, an increase in mental health issues, specifically related to stress, has been widely observed in Canadian engineering students [9], [11]. Time-based stress may be caused from and result in many of the above issues, both directly and via frustration from not being able to effectively employ time management tools and strategies. A negative cycle of stress leading to burnout may begin if students forgo proper sleeping, eating, relaxation, exercise, and other self-care habits as a result of stressors [22], [23]. This cycle of stress has been observed in undergraduate engineering students in recent years, which includes personal experiences of one of the authors.

\subsection{Conceptual Frameworks in ATBDM}

The paper introducing ATBDM outlined social perceptions of time, time management, and decision making abilities (including self-regulated learning) as the basis for ATBDM behaviours [1]. A literature review revealed no existing time management and decision making instruments deemed appropriate for the specifics of the proposed research study [16], [17]. Therefore, a new research instrument was developed based on prior time management instruments, new theories of social time perceptions, and concepts from decision making. This section overviews these concepts and how they were synthesized into the new instrument.

2.2.1. Social perceptions of time. The perception of time has been changing in recent decades. The pace of social time has been notably accelerating, meaning that social interactions, including work, occur at a faster pace, which can have positive and negative consequences [12], [13]. In addition, work-life (by analogy, school-life) and home-life have become increasingly integrated and fragmented, resulting sometimes in social consequences such as decreases in time use efficiency [13], [14]. This new digital era, where people may be expected to be "always on," (that is, often communicating and making decisions relatively quickly and often) may therefore indicate a need to manage time differently [14], [24].

This focus on scheduling and immediacy is not always efficient, as Wajcman noted in her study on overly-scheduled Silicon Valley software engineers [14]. Similarly, Liao et al. identified several types of time experienced by university students including "scheduled" and "timeless" times [12]. One observation was that students complying with schedules sometimes found it hard to enter more "timeless" experiences of time (which may be more beneficial for deep learning and entering a state of "flow"); for example, one student found it difficult to effectively complete homework during 1-hour breaks. This recalls the study suggesting surface learning in engineering students under time pressure, and the students who missed class for personal time [2], [18].

There have been some engineering educators who attempt to combat this rushed-schedule feeling in students by employing meta-cognitive techniques with students [25], [26]. Elsewhere, metacognitive techniques have been shown to be effective in improving attendance and related time-management behaviours, including in students [27]. 
The issue of internet-connected devices may be contributing to poor time management via this highspeed, reaction-type social norm. It may be the case that successful students employ certain strategies to combat these distractions. Those who can employ diverse time strategies may have an advantage in education: it has been suggested that online courses require different time management strategies than conventional lectures [28], [29].

Ultimately, the most effective time management strategies may be person-dependent, situation-dependent, and/or time-dependent. This nuance is not considered in any time-management instrument reviewed to date.

2.2.2. Decision-Making. Decision-making theories used here borrow from the self-regulated learning and self-efficacy literature. The efficacy of deciding both how to spend time and which strategies to employ is important: students will be unlikely to use new strategies they do not trust, especially when under stress.

Self-efficacy, according to Bandura's agency theory, can be affected in one of four ways: social persuasion, vicarious experiences, performance accomplishments, and physiological reactions [30]. The first two of these are social in nature: social persuasion is when one is told what to do from a trusted source, and vicarious experience is when one witnesses another accomplishing something. When it is assumed by conventional wisdom that all strategies will work for different people, these social sources of self-efficacy become potentially insidious: for example, if a student $\mathrm{A}$ takes advice from student B to skip class because it is not worth the time, but student A would have benefitted from going to class.

The performance accomplishments and physiological reactions may also have a role in timebased decisions. For example, having a "rush" when finishing work may encourage that behaviour again (physiological), and using a poor behaviour with moderately good results may discourage students from seeking optimal behaviours for excellent results (because of the risk inherent with changing strategies).

Prochaska, Norcross, and DiClemente's model of enacting personal change and Zimmerman's cyclical model of self-regulation are somewhat conceptually similar in that both models use a cyclical process where first one has a belief or idea is held to be advantageous, followed by intention to act on that idea, and finally actually acting upon it [22], [31]. This dimension of decision-making has not been employed in time management literature to date, although in 2018, Aeon and Aguinis called for an increased study of decisionmaking in time management contexts [15].

CEEA20; Paper 158

Montreal; June 18-20, 2020 -3 of $8-$
2.2.3. Existing Time Management Literature. Several issues have been identified in the current time management literature both by Hellsten and Claessens et al., the most significant of which are reliability issues within the most popular time management instruments, and convergence issues between instruments [16], [17]. In addition, some instruments identified ask questions which are conceptually unrelated to time management, such as whether one is generally organized (not specifically time-organized).

Subsequent issues that have been identified include the lack of consideration for multiple successful strategies, assumption of few over-arching time management behavioural factors, and lack of decisionmaking dimension. The lack of consideration of multiple strategies means that current instruments assume all people in all places at all times can successfully employ the same strategies. As was discussed in the prior section on social perceptions, not all time is perceived in the same way, so it is logically fallacious to necessarily assume all time can be managed in the same way.

This approach also leads to the factors-based approach, which assumes singular underlying factors. In these factor models, generally three to five factors are hypothesized; however, this is not always the case: Hellsten documents one case where Schriber and Gutek identify 13 distinct factors for time at work [17], [32]. Unfortunately, Hellsten did not further consider Schriber and Gutek's findings in her review, and it appears that the possibility of a high-factor model of time management has not been recently revisited in the literature.

A clusters-based approach, where behaviours cluster into groups, may provide a method of grouping people without assuming singular, universally correlated factors [33]. The decision-making dimension, would also be compatible with a cluster analysis, which has already been discussed.

2.2.4. The BIR or "Level" Model of Time Management Behaviours. Having found deficiencies in the current selection of time management theories and survey tools, a new theoretical construct for understanding time management is proposed. It seeks to remedy the current theories by introducing a wide range of behaviours, challenging assumptions about which behaviours (or groupings of behaviour) are "good," and incorporating three levels of decision making: Belief, Ideals, and Realities (BIR). The incorporation of all three levels is expected to offer more insight into which behaviours students believe are useful, which they wish to exhibit, and which they are currently employing. Tension between these levels may be a source of stress and/or inefficient or ineffective use of time.

The multiple behaviours (approximately fourteenas some may or may not be separate) were initially sourced from cross-referencing a multitude of existing 
time management papers and meta-analyses. Several behaviours, such as planning and scheduling, were separated because some studies would amalgamate them while others would consider them separately.

The BIR model also supposes time, place, and person dependency on the behaviours. That is, different times, places, and people will require employment of different behaviours. A full description of the conceptualization and development process for the BIR model will be published at a later date.

\section{Pilot Study}

Pilot studies are used to ensure the validity of the study instrument and relevance of theory, and allow for improvements before investing resources in a full study. Pilot studies of this nature have been successfully employed in past studies on a similar population [34], [35]. This pilot study involved the creation of a survey instrument, the recruitment of graduate engineering students as a test population, and the use of surveys and interviews to align theory with instrument design while improving both. Institutional ethics approval was received before the pilot study phase was implemented.

\subsection{Survey Instrument and Interview Script Design \& Development}

Elements in the pilot surveys and follow-up interviews were designed for the dual purposes of testing the survey instrument for completeness, reliability, validity, interpretability, and user-experience, while also testing the theoretical direction for familiarity, completeness, and precision.

3.1.1. Survey Tool Creation. The survey comprised of five different sections in the order of (1) Ideal/Realitylevel behaviours (IR), (2) Belief-level behaviours (B), (3) academics, success \& burnout, (4) commitments, and (5) demographics. Approximately $60 \%$ of the survey by time was for BIR questions, and $40 \%$ for other questions. It was designed to be completed in 15-20 minutes.

Pilot study demographic questions focused on program, discipline, age, years of school, international status, and professional (including internship) experience; academics focused on perceived success, GPA, absenteeism from class, and hours of class; commitments and stressors had a general burnout measure, sources of academic pressure (family, peers, self, etc.), and extracurricular commitments.

The IR-level behaviours were mostly two-item Likert scales (i.e. for each behaviour, two questions at the Ideal-level, and two questions at the Reality-level). Although two-item scales are generally not recommended, they were in this case the preferred risk over an overly-long survey [36]. This was justified because of the simplicity of the questions and the exploratory nature of the work, for which covering a large basis of behaviours was prioritized.

Reasons for missing or being late to class, as well as types of stressors, were amalgamated from literature and experiential observations. Survey questions on student burnout and engagement were taken directly from existing literature [37].

The survey also contained a user-generated code that was used to select (using striated sampling) a wide range of interviewees. The full process for keeping data confidential while using such a code was complex, and was approved by the institutional ethics board before implementation of the pilot study.

Finally, extra open-ended textboxes were included at the bottom of each page to give an additional feedback opportunity on question clarity. As an unexpected benefit, in some cases the feedback also helped improve theory completeness.

3.1.2. Follow-up Interview Guide. The interview guide consisted of a survey think-aloud (the largest section), questions on undergraduate time management, and questions covering theory completeness.

Previous studies have used the think-aloud process, where participants read and interpret the survey questions aloud, to find errors in interpretation and survey design [34], [35]. During the think-aloud, follow-up questions were used to test the categorization of some topics, for example whether the selected categories for "reasons to be late or absent from class" formed a valid basis (i.e. whether each category was unique and independent). Questions about user experience, such as scale design (e.g. the functionality of agree-disagree vs. alwaysnever) were also posed.

After the think-aloud portion, the interview briefly investigated participants' perspectives on undergraduate time management, both first-hand (i.e. their own past), and second-hand (e.g. as an observing TA). These differences in past and present experience were also used to explore any generational changes which may exist.

Finally, to ensure theory completeness, a short section of questions was used to test the relative importance of unasked but possibly related topics (e.g. sleep, eating habits, and procrastination) by asking participants about experiences in their previous engineering undergraduate program. The interview was designed to last approximately one hour.

\subsection{Methods and Recruitment}

The target population for the pilot study was graduate engineering students (M.A.Sc., M.Eng. and Ph.D.). Graduate students offered several advantages including availability, avoiding contamination of the target population ahead of the full study, and ability to reflect from an external perspective over their entire undergraduate career. At the end of the survey,

CEEA20; Paper 158

Montreal; June 18-20, $2020 \quad-4$ of 8 - 
participants could elect to leave contact information on a separate form to volunteer for the think aloud interviews.

The survey was administered using the institution's survey software. Interviews were done in person in a private room and were recorded with participant consent. The transcripts were hand-typed, and then the original audio files destroyed.

\subsection{Results and Analysis Approach}

Data from twelve full and one partially completed survey were collected, and three think-aloud interviews were conducted. The completed surveys represented a relatively wide range of engineering students (eight M.A.Sc. and four Ph.D., representing five engineering disciplines), however despite targeted recruiting efforts, M.Eng. students were notably absent. Two M.A.Sc. students and one Ph.D. student were then interviewed.

3.3.1. Quantitative Analysis. The quantitative data were analyzed using a variety of techniques. The BIR data was first analyzed for scale reliability. Peters recommends using multiple methods for testing reliability including a correlogram (which combines item histograms, scatterplots, and correlation coefficients into a single efficient plot), several reliability tests, and exploratory factor analysis (EFA) [38]. Eisinga, Grotenhuis, and Pelzer recommend using the SpearmanBrown coefficient for 2-item scales, although they do not themselves endorse using 2-item scales [36]. In addition, a cluster analysis in $\mathrm{R}$ used "heatmap" to produce a heatmap-dendogram hybrid. For the BIR data, these tests were then analyzed with the qualitative think-aloud data, described in the mixed-methods section below.

Although the burnout and engagement scales were tested using Cronbach's alpha and EFA and found to be reliable, an updated version of the scales was made available in the reference literature and subsequently substituted for the main study. The other sections of the survey were assessed qualitatively, as the categorical, opt-in nature of those questions meant that there were insufficient number of participants from which to draw statistical conclusions.

3.3.2. Qualitative Analysis. The qualitative data consisted of the interview transcripts, the extra textbox data from the bottom of each survey page, and notes taken during the interviews. Before being used for analysis, the transcripts were compared question-by-question and similarities between interviewees were noted.

All three sources were used to improve the validity of the divisions for absenteeism, commitments, and stressors. For example, a distinction was made between sleeping through a class by accident and on purpose - as a direct result of the pilot study analysis, these are now grouped in separate categories.

CEEA20; Paper 158

Montreal; June 18-20, 2020
3.3.3. Mixed Methods Analysis. To improve the accuracy of changes in BIR behaviours with relatively few responses and relatively many items, a triangulationstyle mixed-methods approach was used [39]: the previous BIR quantitative and qualitative analyses were compared to one another and to the question wording to holistically assess when a question wording needed modification. By using triangulation, false positives and false negatives from both the surveys and the interviews were reduced, optimizing the number of item changes.

B-, I-, and R-level questions for each behaviour were analyzed together to investigate behaviour-specific errors. Questions that grouped or factored on the wrong levels were immediately modified; questions with minor issues were changed only after a critical re-analysis, since minor issues may have resulted from statistical error or personal preferences. The correlogram and heatmapdendogram were notably useful in interpreting the interand intra-level results quickly and efficiently.

3.3.4. Discussion on Graduate Results. The survey questions were not designed for a graduate student environment, nor were the pilot results intended to be representative (having low participant-to-question ratio). Moreover, the number of modifications made further reduces claims of statistical representation. However, the wide range of both BIR and interview responses seems to indicate the existence of a wide range of successful timemanagement strategies. For example, whereas one participant has always preferred strictly keeping separate working non-working hours, and another has preferred irregular working hours and accomplishing a mix of task types throughout the day, both graduate students had successful undergraduate engineering experiences.

\subsection{Discussion and Instrument Improvements}

A number of modifications were made following the pilot study which are expected to have a significant impact on the reliability, interpretability, validity, userexperience, and comprehensiveness of the full study. These changes encompassed improvements to question wording, response option, layout and user experience, and alignment to theory. Some of the more significant and generally useful improvements are described below.

3.4.1. Question Modifications. Of the 61 IRbehaviour questions, nine were completely re-written, and eighteen underwent substantial changes. Four questions were removed, and six were added, of which four constituted the previously unconsidered behaviour of "separating vs. integrating school and non-school work and activities." Deleted questions were removed because of redundancy and/or unreliability.

As noted previously, scales measuring different types of procrastination and timely engagement (the theoretical opposite) were added based on unanimously 
input from the interviewees. Additional questions were included in the demographics sections to gain a better understanding of specific background in education, more specific internship and other experiences, and other nonschool time. Gender was added as an optional text-entry question. Despite the additions, the overall time was kept to 15-20 minutes because of efficiency improvements in user experience.

3.4.2. Likert Scale Modifications. For the pilot study, most of the Likert scales used were 5-point "mostly agree / somewhat agree / neutral / somewhat disagree / mostly disagree," with the exception being the "late and absenteeism" questions which used a select-all-that-apply scale of "none / late once / late multiple time / absent once / absent multiple times." "Mostly agree" was used to try and increase the resolution of the scale as "Agree" was thought to have a higher threshold for selection.

Although "mostly agree to mostly disagree" worked well for the IR scales, it did not work well for other questions. On the recommendation of an interviewee, question-specific Likert scales such as "almost neveralmost always" were substituted where needed.

One notable change in scale labels and item wording was at the Belief (B) level. The wording of many B-level questions were too positively connoted, skewing the scale towards high scores. More neutral question wording and a six-point Likert scale were substituted to enhance the scale resolution. Early data from the full study seems to indicate these changes have succeeded in reducing skew in the B-level questions.

Perhaps the most important change to the Likert scales was changing "N/A" to the more inviting "Does not apply to me" and adding instructions on when to use it. Pilot interviewees found "N/A" to be impersonal, and therefore were dissuaded from using it even when they claimed it would have been the most appropriate answer.

3.4.3. Additions and Extension. The inquiry into the relative importance of related but excluded variables resulted in the addition of procrastination questions to the questionnaire as well as an optional 5-minute survey extension. Procrastination was universally agreed to be the most important excluded variable, and therefore was added to the main survey. The topics of sleeping and eating habits, weekend versus weekday variations, and previous time management training were relevant enough to warrant the optional extension, which provides some coverage without increasing the main survey length.

3.4.4. User Experience Improvements. The instructions, definitions, and prompts were extensively revised to improve user experience. The concision of the instructions was improved, in part by using bullet points. The definitions were taken from a preface and placed near their related questions. Prompts were added at the bottom of each page to inform the user of their progression through the survey, by listing all survey sections with expected times and checking off the completed sections.

Including data entry boxes at the bottom of each survey page to express additional thoughts was kept as it was effective as both a user-experience and reliability tool. Early indications of the full study indicate this has already proven useful, for example by identifying ADHD as another potential factor in time management and clarifying the reasoning for certain responses.

Finally, an option to be informed of the study results via email was added to the survey because of enthusiasm from the interviewees. Preliminary data shows this is well-received in the full study with a $\sim 40 \%$ opt-in rate.

\subsection{Pilot Conclusions and Lessons Learned}

The pilot study brought substantial improvements to both the survey and the theory of this research project. The new survey has an improved user experience, clearer instructions, and is more comprehensive and reliable. Notably, having optional comment boxes on each page should be useful in keeping information reliable; changing "N/A" to "Does not apply to me" should increase its appropriate use; and adding the option to be contacted when results become available has been well subscribed. Many of these changes were unexpected despite rigorous pre-pilot testing and several reviews; as such, pilot studies are recommended for engineering education research of this nature.

\section{Future Work}

The improved survey instrument was administered to undergraduate engineering students at one institution starting in March 2020. Expanding the survey to other institutions is also being considered.

The final method of quantitative data analysis has not yet been confirmed, however given pilot success, both clustering and factoring approaches will likely be used. If enough participants are recruited, a confirmatory stage will be employed to increase reliability and validity of the findings. Assuming survey responses can be clustered into groups, striated sampling will be used to select a wide variety of interviewees. Interview topics will include the causes, consequences, and range of time management strategies employed by undergraduate students.

\section{Conclusions}

Canadian engineering students appear to be having difficulties in managing their academic time. This may be manifesting itself in a variety of symptoms including absenteeism, higher stress levels, and increase in the surface-learning approach. The source of the difficulties may be personal, institutional, or societal.

Current time management literature appears to have many issues with reliability, convergence, and

CEEA20; Paper 158

Montreal; June 18-20, $2020 \quad-6$ of $8-$ 
comprehensiveness. The new BIR framework seeks to remedy these by assuming many behaviours, incorporating multiple decision-making levels, and using a cluster-based rather than factor-based approach.

A pilot study with graduate engineering students was used to improve the research survey tool. Twelve graduate students completed the survey and three participated in interviews. Numerous modifications to questions, Likert scales, and user experience resulted from the pilot study analysis. Pilot studies are recommended for future studies of a similar nature.

Currently, an ongoing full study is investigating time management in undergraduate engineering students, including the possibility of multiple successful strategies and current barriers to time management success.

\section{Acknowledgements}

The authors would like to thank all the graduate engineering student participants who took the time to complete the survey, and especially those who took the time to do the think-aloud interviews. The authors would also like to thank the Faculty of Engineering and Applied Science at Queen's University for their financial support in this research.

\section{References}

[1] N. J. Rupar and D. S. Strong, "What to do? A Review of the Academic Time-Based DecisionMaking Literature," Proc 2019 Can. Eng. Educ. Assoc. CEEA19 Conf, 2019.

[2] S. Doré, "What Seniors Have to Say About Their Engagement," Proc. Can. Eng. Educ. Assoc. CEEA, Jan. 2017, doi: 10.24908/pceea.v0i0.6475.

[3] E. Kuley, S. Maw, and T. Fonstad, "Understanding Barriers to Student Success: What Students Have to Say," Proc. Can. Eng. Educ. Assoc. CEEA, Jan. 2017, doi: 10.24908/pceea.v0i0.6507.

[4] N. Nelson and R. Brennan, "A Snapshot of Engineering Education in Canada," in Proc. 2018 Canadian Engineering Education Association (CEEA-ACEG18) Conf., 2018.

[5] M. Bunn, A. Bennett, and P. J. Burke, "In the anytime: Flexible time structures, student experience and temporal equity in higher education," Time Soc., p. 0961463X1878764, Jul. 2018, doi: 10.1177/0961463X18787649.

[6] W. Li, R. M. Bennett, T. Olsen, and R. McCord, "Engage Engineering Students in Homework: Attribution of Low Completion and Suggestions for Interventions," Am. J. Eng. Educ., vol. 9, no. 1, pp. 23-38, Jun. 2018.

[7] N. Manjikian, "Reflections on Five Years of Coherent Teaching Accross Three Courses on

CEEA20; Paper 158

Montreal; June 18-20, 2020
Digital Logic and Computer Systems," Proc 2017 Can. Eng. Educ. Assoc. CEEA17 Conf, 2017.

[8] D. M. Smith and S. Maw, "Supplementary Results of the CAIS-1 Survey on Cheating in Undergraduate Engineering Programs in Saskatchewan," p. 7, 2017.

[9] A. D'Entremont, "Being a C-student is the new normal: a literature review on grades, self-worth, and mental wellbeing," Proc. Can. Eng. Educ. Assoc. CEEA, Dec. 2018, doi: 10.24908/pceea.v0i0.13077.

[10] R. Brennan, "A Systematic Review of Canadian Engineering Education Research 2004-2017," Proc. Can. Eng. Educ. Assoc. CEEA, Dec. 2018, doi: 10.24908/pceea.v0i0.13079.

[11] R. Clemmer, K. Gordon, and J. Vale, "Will That Be on the Exam? - Engineering Student Perceptions of Assessments, Memorization, and Approaches to Learning," Proc 2018 Can. Eng. Educ. Assoc. CEEA-ACEG18 Conf, 2018.

[12] T. F. Liao, J. Beckman, E. Marzolph, C. Riederer, J. Sayler, and L. Schmelkin, "The social definition of time for university students," Time Soc., vol. 22, no. 1, pp. 119-151, Mar. 2013, doi: 10.1177/0961463X11404385.

[13] M. Huijer, "Book Review: Pressed for time: The acceleration of life in digital capitalism by Judy Wajcman," Time Soc., p. 0961463X1775260, Jan. 2018, doi: 10.1177/0961463X17752601.

[14] J. Wajcman, "The Digital Architecture of Time Management," Sci. Technol. Hum. Values, vol. 44, no. 2, pp. 315-337, Mar. 2019, doi: 10.1177/0162243918795041.

[15] B. Aeon and H. Aguinis, "It's About Time: New Perspectives and Insights on Time Management," Acad. Manag. Perspect., vol. 31, no. 4, pp. 309330, Nov. 2017, doi: 10.5465/amp.2016.0166.

[16] B. J. C. Claessens, W. van Eerde, C. G. Rutte, and R. A. Roe, "A review of the time management literature," Pers. Rev., vol. 36, no. 2, pp. 255-276, Feb. 2007, doi: 10.1108/00483480710726136.

[17] L.-A. M. Hellsten, "What Do We Know About Time Management? A Review of the Literature and a Psychometric Critique of Instruments Assessing Time Management," Time Manag., Mar. 2012, doi: 10.5772/37248.

[18] R. McCord and H. M. Matusovich, "Study Context Matters: A Case Study on How Time Crunches Lead to Coping Modes of Learning," presented at the 2017 ASEE Annual Conference \& Exposition, Jun. 2017, Accessed: Jan. 10, 2019. [Online]. Available: https://peer.asee.org/study-contextmatters-a-case-study-on-how-time-crunches-leadto-coping-modes-of-learning.

[19] P. Dumond, "A Course Based Approach to Recognizing Student Efforst in Engineering 
Design Competitions," Proc 2017 Can. Eng. Educ. Assoc. CEEA17 Conf, p. 7, 2017.

[20] K. M. Greene and J. L. Maggs, "Revisiting the Time Trade-off Hypothesis: Work, Organized Activities, and Academics during College," $J$. Youth Adolesc., vol. 44, no. 8, pp. 1623-1637, Aug. 2015, doi: 10.1007/s10964-014-0215-7.

[21] C. E. Goodson, S. L. Miertschin, and B. L. Stewart, "Design of Online Courses: Implications for Student Time Management," presented at the 2014 ASEE Annual Conference \& Exposition, Jun. 2014, pp. 24.372.1-24.372.16, Accessed: Oct. 01, 2018. [Online]. Available: https://peer.asee.org/design-of-online-coursesimplications-for-student-time-management.

[22] M. McNamara and T. Straathof, Coping strategies to promote occupational engagement and recovery: a program manual for occupational therapists and other care providers. 2017.

[23] World Health Organization, "Burn-out an 'occupational phenomenon': International Classification of Diseases," WHO. http://www.who.int/mental_health/evidence/burnout/en/ (accessed Dec. 16, 2019).

[24] A. Johri, H. J. Teo, J. Lo, M. Dufour, and A. Schram, "Millennial engineers: Digital media and information ecology of engineering students," Comput. Hum. Behav., vol. 33, pp. 286-301, Apr. 2014, doi: 10.1016/j.chb.2013.01.048.

[25] G. J. Evans, "A Windmills of Your Mind: Metacognition and Lifelong Learning," in Proc. 2018 Canadian Engineering Education Association (CEEA-ACEG18) Conf., 2018.

[26] A. Maleki, C. Picolo, and J. Verrett, "Effect of a Mini Lesson on Self-Regulated Learning on Students' Learning," in Proc. 2018 Canadian Engineering Education Association (CEEA18) Conf., 2018.

[27] G. Oettingen, H. B. Kappes, K. B. Guttenberg, and P. M. Gollwitzer, "Self-regulation of time management: Mental contrasting with implementation intentions," Eur. J. Soc. Psychol., vol. 45, no. 2, pp. 218-229, Mar. 2015, doi: 10.1002/ejsp.2090.

[28] A. Pardo, F. Han, and R. A. Ellis, "Exploring the relation between self-regulation, online activities, and academic performance: a case study," in Proceedings of the Sixth International Conference on Learning Analytics \& Knowledge - LAK '16, Edinburgh, United Kingdom, 2016, pp. 422-429, doi: $10.1145 / 2883851.2883883$.

[29] S. L. Miertschin, C. E. Goodson, and B. L. Stewart, "Managing Time in Online Courses: Student Perceptions," presented at the 2012 ASEE Annual Conference \& Exposition, Jun. 2012, pp. 25.911.125.911.14, Accessed: Oct. 01, 2018. [Online].
Available: https://peer.asee.org/managing-time-inonline-courses-student-perceptions.

[30] K. Bartimote-Aufflick, A. Bridgeman, R. Walker, M. Sharma, and L. Smith, "The study, evaluation, and improvement of university student selfefficacy," Stud. High. Educ., vol. 41, no. 11, pp. 1918-1942, Nov. 2016, doi: 10.1080/03075079.2014.999319.

[31] M. Puustinen and L. Pulkkinen, "Models of Selfregulated Learning: A review," Scand. J. Educ. Res., vol. 45, no. 3, pp. 269-286, Sep. 2001, doi: 10.1080/00313830120074206.

[32] J. B. Schriber and B. A. Gutek, "Some time dimensions of work: Measurement of an underlying aspect of organization culture.," $J$. Appl. Psychol., vol. 72, no. 4, p. 642, Mar. 1988, doi: 10.1037/0021-9010.72.4.642.

[33] J. Chasmar and K. M. Ehlert, "Cluster Analysis Methods and Future Time Perspective Groups of Second-Year Engineering Students in a MajorRequired Course," presented at the 2018 ASEE Annual Conference \& Exposition, Jun. 2018, Accessed: Jan. 10, 2019. [Online]. Available: https://peer.asee.org/cluster-analysis-methodsand-future-time-perspective-groups-of-secondyear-engineering-students-in-a-major-requiredcourse.

[34] H. Smith and D. Strong, "Creative Confidence and the Arts: Measuring a Potential Contributing Factor to Students' Motivation to Engage in Engineering Creativity," Proc. Can. Eng. Educ. Assoc. CEEA, Dec. 2018, doi: 10.24908/pceea.v0i0.13071.

[35] N. Lanziner and D. S. Strong, "Measuring Students' Motivation to Engage in Sustainable Engineering Practice," Proc. Can. Eng. Educ. Assoc. CEEA, 2017, doi: 10.24908/pceea.v0i0.10197.

[36] R. Eisinga, M. te Grotenhuis, and B. Pelzer, "The reliability of a two-item scale: Pearson, Cronbach, or Spearman-Brown?," Int. J. Public Health, vol. 58, no. 4, pp. 637-642, Aug. 2013, doi: 10.1007/s00038-012-0416-3.

[37] W. B. Schaufeli, I. M. Martínez, A. M. Pinto, M. Salanova, and A. B. Bakker, "Burnout and Engagement in University Students: A CrossNational Study," J. Cross-Cult. Psychol., vol. 33, no. 5, pp. 464-481, Sep. 2002, doi: $10.1177 / 0022022102033005003$.

[38] G.-J. Y. Peters, "The alpha and the omega of scale reliability and validity: why and how to abandon Cronbach's alpha and the route towards more comprehensive assessment of scale quality," doi: 10.31234/osf.io/h47fv.

[39] J. W. Creswell, A concise introduction to mixed methods research. 2015. 